\title{
Widespread dissemination of extended- spectrum $\beta$-lactamase-producing, multidrug-resistant Escherichia coli in livestock and fishery products in Vietnam
}

Ha Viet Le', Ryuji Kawahara², Diep Thi Khong ${ }^{1}$, Hoa Thi Tran ${ }^{1}$, Thang Nam Nguyen ${ }^{1}$, Khai Ngoc Pham', Michio Jinnai ${ }^{2}$, Yuko Kumeda ${ }^{2}$, Tatsuya Nakayama ${ }^{3}$, Shuhei Ueda ${ }^{3}$ and Yoshimasa Yamamoto ${ }^{2,3^{*}}$

\begin{abstract}
Background: Widespread dissemination of extended-spectrum $\beta$-lactamase (ESBL)-producing bacteria is a threat to public health. Since livestock products are possible reservoirs of ESBL-producing bacteria, food-borne dissemination of ESBL-producing bacteria and the characteristics of such organisms in food products should be assessed to evaluate potential sources of contamination.

Results: A total of 253 food samples from poultry, pork, shrimp, and fish were collected from local markets in a rural area of Vietnam from 2013 to 2014. ESBL-producing Escherichia coli were isolated from these samples, and their ESBL genotypes, phylogenetic groups, and antibiotic resistance profiles were assessed. Overall, a high percentage (68.4\%) of the food samples was contaminated with ESBL-producing E. coli, and samples from livestock and fishery products showed similar levels of contamination. The ESBL-producing $E$. coli isolated from the food samples harbored genes of the CTX-M-1, CTX-M-9, and TEM groups. Analysis of the antibiotic resistance profiles of the ESBL-producing E. coli isolates from the food samples showed a high degree of multidrug resistance. The prevalence of extensively multidrug-resistant ESBL-producing $E$. coli that were resistant to at least five antibiotic drug classes in poultry, pork, shrimp, and fish samples, was $92.1,69,56.5$, and $62.5 \%$, respectively.

Conclusions: The results of this study confirmed the widespread dissemination of ESBL-producing E. coli in both livestock and fishery products from a rural area in Vietnam. The high prevalence of extensively multidrug-resistant ESBL-producing E. coli in food products highlights the importance of continuous monitoring of food products for the presence of these bacteria, particularly in underdeveloped countries.
\end{abstract}

Keywords: Antibiotic resistance, Extended-spectrum $\beta$-lactamase (ESBL), Escherichia coli, Livestock products, Vietnam

\section{Background}

Antimicrobial drug resistance is a growing public health concern worldwide (Hawkey and Jones 2009), and in particular, the increasing prevalence of extendedspectrum $\beta$-lactamase (ESBL)-producing bacteria is an emerging threat to public health (Pitout and Laupland 2008; Woerther et al. 2013). In the 1980s and 1990s, ESBL-producing bacteria, mainly SHV and TEM types,

\footnotetext{
* Correspondence: yyamamot@iph.pref.osaka.jp

${ }^{2}$ Osaka Prefectural Institute of Public Health, Osaka, Japan

${ }^{3}$ Osaka University Global Collaboration Center, 2-7 Yamadaoka, Suita, Osaka

565-0871, Japan

Full list of author information is available at the end of the article
}

were almost exclusively detected in nosocomial settings (Woerther et al. 2013). However, in recent years, prevalence of the CTX-M-type ESBL-producing bacteria has increased in both nosocomial and community settings (Bush and Jacoby 2010). Furthermore, ESBL-producing bacteria often exhibit co-resistance to multiple classes of antibiotics (Coque et al. 2008), which may increase the risk of poor clinical outcomes because of the lack of effective treatment options.

Recent studies indicate that the prevalence of ESBLproducing bacteria in developing countries, especially Asian countries, has increased to more than $70 \%$ of 
community residents (Tian et al. 2008; Coque et al. 2008; Sasaki et al. 2010; Luvsansharav et al. 2012; Nakayama et al. 2015). Food and food-producing animals are considered a potential reservoir of these bacteria (Geser et al. 2012; Liebana et al. 2013). Although limited studies on the prevalence of ESBL-producing bacteria have been conducted in Asian countries, the results show widespread dissemination of these bacteria in the community (Sasaki et al. 2010; Nakayama et al. 2015).

The present study determined the prevalence, genetic and phylogenetic groups, and antibiotic resistance profiles of ESBL-producing Escherichia coli (ESBL-E. coli) in retail livestock and fishery products in Vietnam.

\section{Methods}

\section{Sample collection and bacterial isolation}

A total of 253 food samples of poultry (chops; $n=60$ ), pork (chops; $n=69$ ), shrimp (freshwater shrimp; $n=60$ ), and fish (ray-finned fish, Southeast Asian tropical freshwater fish, Asian carp, snakehead fish, and tilapia; $n=64$ ) were purchased from three local markets in a rural area of Thai Binh, Vietnam from June 2013 to December 2014. For bacterial isolation, $25 \mathrm{~g}$ of each food sample was inoculated into $225 \mathrm{~mL}$ of buffered peptone water and incubated at $37{ }^{\circ} \mathrm{C}$ for $18 \mathrm{~h}$. The cultures were then inoculated on CHROMagar ECC medium (CHROMagar, Paris, France) containing $1 \mu \mathrm{g} / \mathrm{mL}$ cefotaxime and cultured at $37{ }^{\circ} \mathrm{C}$ for $24 \mathrm{~h}$. One to three colonies that showed the characteristics of $E$. coli were collected from each sample plate.

\section{Screening and identification of ESBL-producing bacteria}

ESBL production by the bacterial isolates was evaluated by the disc diffusion method on Mueller-Hinton agar plates (Oxoid, Cheshire, UK) using ceftazidime and cefotaxime with and without clavulanic acid as recommended by the Clinical and Laboratory Standards Institute (Clinical and Laboratory Standards Institute 2013). ESBL-producing isolates were identified as E. coli by the IMViC test (Thampuran et al. 2005).

\section{Genotyping of ESBL-encoding genes}

Polymerase chain reaction (PCR) was used to detect genes encoding ESBLs. Bacterial DNA was extracted by boiling the bacterial suspension in tris(hydroxymethyl)aminomethane-EDTA buffer ( $\mathrm{pH}$ 8.0). The extracted DNA was used as the template for amplification by multiplex PCR using primers specific for genes encoding enzymes belonging to the TEM, SHV, CTX-M-1, CTX-M-2, CTX-M-9, and CTX-M-8/CTX-M-25 groups (Le et al. 2015). The PCR conditions used were as follows: initial denaturation at $95{ }^{\circ} \mathrm{C}$ for $5 \mathrm{~min}$, followed by 25 cycles of denaturation at $95^{\circ} \mathrm{C}$ for $30 \mathrm{~s}$, annealing at $60{ }^{\circ} \mathrm{C}$ for $90 \mathrm{~s}$, and extension at $72{ }^{\circ} \mathrm{C}$ for $90 \mathrm{~s}$, with a final extension at $68{ }^{\circ} \mathrm{C}$ for
$10 \mathrm{~min}$. The PCR products were visualized by electrophoresis on a $2 \%$ agarose gel followed by ethidium bromide staining.

\section{Determination of phylogenetic groups}

The phylogenetic groups of ESBL-E. coli were identified by multiplex PCR amplification of a combination of two genes, $\operatorname{ch} u A$ and $y j a A$, and a DNA fragment, TspE4C2, as described previously (Chen et al. 2014).

\section{Antimicrobial susceptibility testing}

Antibiotics from seven groups, namely, $\beta$-lactams (ampicillin [AMP], cefoxitin [FOX], cefotaxime [CTX], ceftazidime [CAZ], and meropenem [MEM]), quinolones (nalidixic acid [NAL] and ciprofloxacin [CIP]), aminoglycosides (kanamycin [KAN], streptomycin [STR], and gentamicin [GEN]), tetracycline (TET), fosfomycin (FOF), phenicols (chloramphenicol [CHL]), and folic acid inhibitors (trimethoprim-sulfamethoxazole [SXT]), were used to test the antimicrobial susceptibility of the isolated ESBL-E. coli strains. Antibiotic susceptibility was determined by the disc diffusion method using antibiotic disks (Becton Dickinson) according to the standard CLSI protocol. The results of the susceptibility tests were interpreted using CLSI document M100-S23 (Clinical and Laboratory Standards Institute 2013).

\section{Statistical analysis}

Statistical analysis was performed using the chi-square test, and the significance level was set at $p<0.05$.

\section{Results}

Prevalence of ESBL-E. coli in food samples

A high percentage $(68.4 \%)$ of the tested poultry, pork, shrimp, and fish samples was contaminated with ESBL-E. coli (Table 1). The prevalence of ESBL-E. coli in the tested samples varied significantly from $50.7 \%$ in pork to $88.3 \%$ in poultry $(p<0.001)$. Samples from livestock and fishery products showed similar prevalence of ESBL-E. coli $(69.5 \%$ in livestock products and $68.8 \%$ in fishery products).

\section{Genotypes of ESBL-E. coli}

Genotype analysis showed that the majority of the ESBL-E. coli isolates belonged to the CTX-M group (359/372 [96.5\%]). Approximately $40 \%$ of the ESBL-E. coli isolates (149/372) belonged exclusively to the CTX-M group, whereas only $3.5 \%$ (13/372) belonged only to the TEM group.

The CTX-M ESBL-E. coli isolates from the food samples belonged to the CTX-M-1 and CTX-M-9 groups (Table 2). The majority of the ESBL-E. coli isolates $(57.3 \%)$ encoded genes for multiple ESBLs, including those of the CTX-M-9, CTX-M-1, and TEM groups. 
Table 1 Prevalence of antibiotic-resistant ESBL-E. coli in the food samples

\begin{tabular}{|c|c|c|c|c|c|}
\hline & \multirow[b]{3}{*}{ Total } & \multicolumn{4}{|l|}{ Food type } \\
\hline & & \multicolumn{2}{|c|}{ Animal products } & \multicolumn{2}{|c|}{ Fishery products } \\
\hline & & Poultry & Pork & Shrimp & Fish \\
\hline No. of samples tested & 253 & 60 & 69 & 60 & 64 \\
\hline No. of ESBL-E. coli-positive samples & $173(68.4 \%)$ & $53(88.3 \%)^{*}$ & $35(50.7 \%)$ & $45(75.0 \%)$ & $40(62.5 \%)$ \\
\hline ESBL-E. coli isolates ${ }^{a}$ & 372 & 126 & 81 & 85 & 80 \\
\hline
\end{tabular}

${ }^{*} p<0.001$, significantly different from the other food type

${ }^{a}$ One to three representative colonies were isolated from each sample

The ESBL-E. coli isolates from poultry samples possessed a higher number of genes encoding multiple ESBLs than the isolates from other food samples (75.4\%, $p<0.001)$. Overall, $32.3 \%$ of the ESBL-E. coli isolates obtained from the food samples encoded genes in the CTX-M-9 and TEM groups, whereas $23.9 \%$ of the ESBL-E. coli isolates encoded genes of the CTX-M-1 and TEM groups. In contrast, only $0.8 \%$ of the ESBL-E. coli isolates only encoded genes of the CTX-M group, which is significantly lower than the $56.5 \%$ of the ESBL$E$. coli isolates that encoded genes in both the CTX-M and TEM groups.

\section{Phylogenetic groups of ESBL-E. coli}

Phylogenetic analysis showed some similarities among all the ESBL-E. coli isolates from the food samples (Table 3). The prevalence of ESBL-E. coli isolates belonging to group B2, which includes virulent clonal groups of human extraintestinal pathogenic E. coli that cause a variety of infections outside of the gastric intestinal tract (Jakobsen et al. 2010), was low in the food samples tested in this study.

\section{Multidrug resistance in ESBL-E. coli}

The antibiotic resistance profiles of the ESBL-E. coli isolates from the food samples tested in this study are shown in Table 4. It should be noted that one ESBL-E. coli isolate from pork sample was resistant to MEM. In addition, $5.4 \%$ of the isolates were resistant to FOX. FOF resistance was very limited, except in isolates from poultry (37.3\%). In addition, the ESBL-E. coli isolates from poultry samples showed significantly higher resistance to KAN, GEN, CIP, NAL, CHL, and FOF than those isolated from the other food samples $(p<0.05)$. Furthermore, $80-81 \%$ of the ESBL-E. coli isolates from poultry, pork, and shrimp were resistant to SXT, compared to only $19 \%$ of the ESBL-E. coli isolates from fish $(p<0.01)$.

A high level of multidrug resistance (MDR), which is defined as resistance to at least one antibiotic drug in three or more antibiotic classes (Cantón and RuizGarbajosa 2011), was observed in all food sources (Fig. 1). Notably, extensive MDR, which is defined as resistance to at least five antibiotic drug classes, was observed in $92.1 \%$ (116/126), 69 \% (56/81), $56.5 \%$ (48/85), and $62.5 \%(50 / 80)$ of the ESBL-E. coli isolates from

Table 2 Prevalence of multiple ESBL-encoding genes in ESBL-E. coli isolated from the food samples tested in this study

\begin{tabular}{|c|c|c|c|c|c|c|c|c|c|c|}
\hline & \multirow{2}{*}{ ESBL genotype } & \multirow{2}{*}{ Total } & \multicolumn{4}{|c|}{ Animal Products } & \multicolumn{4}{|c|}{ Fishery Products } \\
\hline & & & \multicolumn{2}{|c|}{ Poultry } & \multicolumn{2}{|c|}{ Pork } & \multicolumn{2}{|c|}{ Shrimp } & \multicolumn{2}{|r|}{ Fish } \\
\hline \multirow{4}{*}{ Multi- ESBL genes } & CTX-M-9/CTX-M-1/TEM ${ }^{a}$ & $\begin{array}{l}1 \\
(0.3 \%)\end{array}$ & \multirow{4}{*}{$\begin{array}{l}95^{\mathrm{b}} \\
(75.4 \%)^{\mathrm{c}}\end{array}$} & -0 & \multirow{4}{*}{$\begin{array}{l}43 \\
(53.1 \%)\end{array}$} & 0 & \multirow{4}{*}{$\begin{array}{l}35 \\
(41.2 \%)\end{array}$} & {$[0$} & \multirow{4}{*}{$\begin{array}{l}40 \\
(50 \%)\end{array}$} & {$[1(1.3 \%)$} \\
\hline & CTX-M-9/CTX-M-1 & 3 & & $1(0.8 \%)$ & & $1(1.2 \%)$ & & 0 & & $1(1.3 \%)$ \\
\hline & CTX-M-9/TEM & $\begin{array}{l}120 \\
(32.3 \%)\end{array}$ & & $55(43.7 \%)$ & & $17(21 \%)$ & & $22(25.9 \%)$ & & $26(32.5 \%)$ \\
\hline & CTX-M-1/TEM & $\begin{array}{l}89 \\
(23.9 \%)\end{array}$ & & $-39(31.0 \%)$ & & $25(31 \%)$ & & L $13(15.3 \%)$ & & L $12(15.0 \%)$ \\
\hline \multirow{3}{*}{ Single-ESBL genes } & CTX-M-9 & $\begin{array}{l}102 \\
(27.4 \%)\end{array}$ & \multirow{3}{*}{$\begin{array}{l}31 \\
(24.6 \%)\end{array}$} & $18(14.3 \%)$ & \multirow{3}{*}{$\begin{array}{l}38 \\
(46.9 \%)\end{array}$} & $\begin{array}{l}26 \\
(32.1 \%)\end{array}$ & & $-35(41.2 \%)$ & \multirow{3}{*}{$\begin{array}{l}40 \\
(50 \%)\end{array}$} & $23(28.8 \%)$ \\
\hline & CTX-M-1 & $\begin{array}{l}44 \\
(11.8 \%)\end{array}$ & & $10(7.9 \%)$ & & $\begin{array}{l}9 \\
(11.1 \%)\end{array}$ & $\begin{array}{l}50 \\
(58.8 \%)\end{array}$ & $9(10.6 \%)$ & & $16(20.0 \%)$ \\
\hline & TEM & $\begin{array}{l}13 \\
(3.5 \%)\end{array}$ & & $-3(2.4 \%)$ & & $3(3.7 \%)$ & & $-6(7.1 \%)$ & & $1(1.3 \%)$ \\
\hline Total & & 372 & 126 & 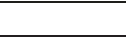 & 81 & & 85 & & 80 & \\
\hline
\end{tabular}


Table 3 Phylogenetic groups of ESBL-E. coli

\begin{tabular}{llllll}
\hline \multirow{2}{*}{ Food type } & Total & A & B1 & B2 & D \\
\cline { 3 - 6 } Poultry & 126 & $42(33.3 \%)$ & $37(29.3 \%)$ & $9(7.1 \%)$ & $38(30.2 \%)$ \\
Pork & 81 & $31(38.3 \%)$ & $25(30.9 \%)$ & $6(7.4 \%)$ & $19(23.5 \%)$ \\
Shrimp & 85 & $28(32.9 \%)$ & $28(32.9 \%)$ & $10(11.8 \%)$ & $19(22.4 \%)$ \\
Fish & 80 & $20(25 \%)$ & $34(42.5 \%)$ & $7(8.8 \%)$ & $19(23.8 \%)$ \\
\hline
\end{tabular}

poultry, pork, shrimp, and fish, respectively. In addition, the ESBL-E. coli isolates from livestock products showed significantly higher MDR than isolates from fishery products $(p<0.05$; Fig. 1$)$.

\section{Discussion}

A previous study conducted in Nha Trang, a mediumsized coastal city in southern Vietnam, showed that the prevalence of ESBL-E. coli in poultry, pork, and shrimp products was $58.7,32$, and $18.3 \%$, respectively (Le et al. 2015). The results of both the present and previous studies showed a higher prevalence of ESBL-E. coli in poultry products than in other food products in both areas of Vietnam. In addition, the results also showed that the overall prevalence of ESBL-E. coli is higher in Thai Binh than in Nha Trang. A study conducted in Denmark reported that $36 \%$ of samples from imported broiler chicken were contaminated with extendedspectrum cephalosporinase-producing E. coli (Agersø et al. 2012), while a similar study conducted in the Netherlands showed that 76.8-94 \% of poultry products were contaminated with ESBL-E. coli (Leverstein-van Hall et al. 2011; Overdevest et al. 2011). Thus, the prevalence of ESBLproducing bacteria in food products varies in different geographical areas.

Southeast Asian countries commonly use an integrated recycling farm system called VAC (vegetable, aquaculture, and caged animal) for food production. This system is effective for small-scale food animal breeding and agricultural production. Stool and waste from humans, animals, and plants are mixed into the soil or put into ponds as food for fish. In this system, antibiotic-resistant bacteria from improperly treated waste could easily enter the ecological cycle, especially in aquatic environments either directly or via sewage ditches. Unhygienic food-handling practices at retail markets may also contribute to the prevalence of antibiotic-resistant bacteria in livestock products. Notably, residual antibiotics are commonly detected in aquatic environments in Vietnam (Hoa et al. 2011). The heavy use of antibiotics in this area can lead to antibiotic contamination of the environment, which likely promotes the development of antibiotic-resistant bacteria (Suzuki and Hoa 2012). In Thai Binh, a typical rural area in northern Vietnam, retail poultry products from different chicken farms are brought to retailers' houses where they are processed before being sold in markets. In addition, most retail pork products originate from households in the community. Nearly $100 \%$ of the freshwater shrimp products are from small domestic rivers near fields or from private pools next to sewage ditches. Almost all fish products are from household fish pools, domestic rivers, or rice fields. Therefore, differences among the sources of the food products and the environments in which they are processed may be the main reasons for the variations in the prevalence of ESBL-E. coli in food samples tested from different geographical areas.

Our results showed that $96.5 \%$ of the ESBL-E. coli isolates encoded genes of the CTX-M group with or without TEM-encoding genes, suggesting the emergence of the CTX-M gene group as the main contributor to the antibiotic resistance of ESBL-E. coli in food, foodproducing animals, healthy people, and patients not only in developed countries but also in underdeveloped countries (Sasaki et al. 2010; Overdevest et al. 2011; Börjesson et al. 2013; Chen et al. 2014; Nakayama et al. 2015).

CTX-M-1 and CTX-M-9 (with or without TEM) were the major subgroups of CTX-M genotypes detected in the ESBL-E. coli isolates from the food samples tested in this study. The results of this study are similar to those of previous studies conducted in other regions of Vietnam (Cortés et al. 2010; Leverstein-van Hall et al. 2011; Le et al. 2015). There was a significant difference in the multi-ESBL genes

Table 4 Percentage of ESBL-E. coli isolated from poultry, pork, shrimp, and fish samples exhibited resistance to antimicrobial agents

\begin{tabular}{|c|c|c|c|c|c|c|c|c|c|c|c|c|c|c|}
\hline \multirow[t]{3}{*}{ Food type } & \multicolumn{14}{|c|}{$\%$ resistance to } \\
\hline & \multicolumn{5}{|c|}{$\beta$ - lactam } & \multicolumn{3}{|c|}{ Aminoglycosides } & \multicolumn{2}{|c|}{ Quinolones } & \multirow{2}{*}{$\begin{array}{l}\text { Tetracycline } \\
\text { TET }\end{array}$} & \multirow{2}{*}{$\begin{array}{l}\text { Phenicols } \\
\mathrm{CHL}\end{array}$} & \multirow{2}{*}{$\begin{array}{l}\text { Phosphomycins } \\
\text { FOF }\end{array}$} & \multirow{2}{*}{$\begin{array}{l}\text { Folic acid inhibitors } \\
\text { SXT }\end{array}$} \\
\hline & $\overline{A M P}$ & FOX & CTX & CAZ & MEM & STR & KAN & GEN & $\overline{C I P}$ & NAL & & & & \\
\hline Poultry (126) & 100.0 & 5.6 & 99.2 & 21.4 & 0.0 & 94.4 & 63.5 & 54.0 & 58.7 & 90.0 & 99.2 & 90.0 & 37.3 & 81.0 \\
\hline Pork (81) & 100.0 & 6.2 & 100.0 & 29.6 & 1.2 & 79.0 & 39.5 & 42.0 & 51.9 & 65.0 & 85.2 & 69.0 & 8.6 & 79.0 \\
\hline Shrimp (85) & 100.0 & 5.9 & 100.0 & 22.4 & 0.0 & 71.8 & 27.1 & 31.8 & 28.2 & 46.0 & 83.5 & 56.0 & 4.7 & 80.0 \\
\hline Fish (80) & 100.0 & 3.8 & 98.8 & 17.5 & 0.0 & 70.0 & 32.5 & 30.0 & 28.8 & 56.0 & 87.5 & 56.0 & 10.0 & $19 . .0$ \\
\hline Total (327) & 100.0 & 5.4 & 99.5 & 22.6 & 0.3 & 80.6 & 43.3 & 41.1 & 43.8 & 67.0 & 90.1 & 71.0 & 17.7 & 67.0 \\
\hline$p$ & & & & & & & $<0.01$ & $<0.05$ & $<0.01$ & $<0.01$ & & $<0.01$ & $<0.01$ & $<0.01$ \\
\hline
\end{tabular}

Number in parentheses indicates the number of isolates 


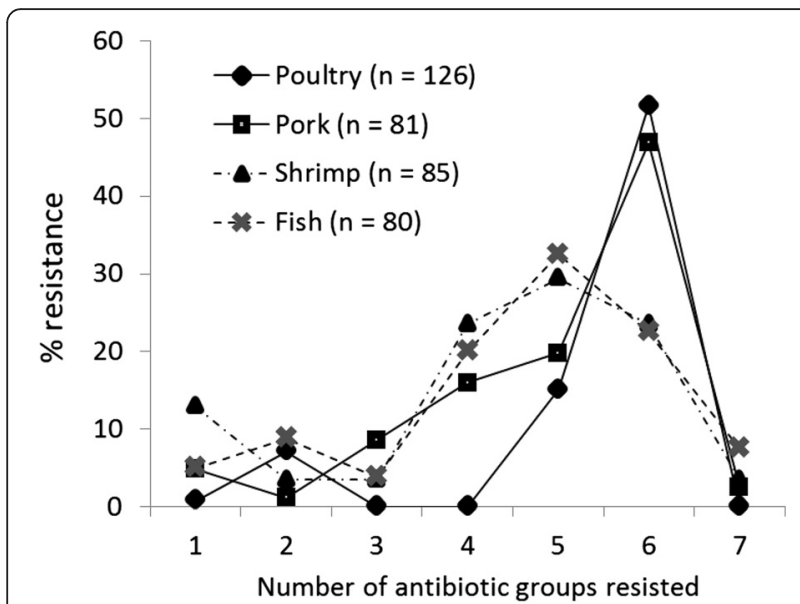

Fig. 1 Degree of MDR ESBL-E. coli isolated from each food sample

encoded by the isolates obtained from poultry compared to those encoded by isolates from pork, shrimp, and fish samples $(p<0.001)$. Antibiotic usage at poultry farms, particularly the long-term antibiotic treatment from hatching to grow-out, may contribute to the acquisition of multiESBL genes, leading to a high prevalence of ESBL-E. coli in poultry products.

ESBL-E. coli belonging to phylogenetic groups A, B1, $B 2$, and D were present in the tested food samples, and there were no significant differences among the food sources. ESBL-E. coli belonging to groups B1 and A accounted for approximately two-thirds of all ESBL-E. coli isolates from the food samples, followed by those belonging to group D. In contrast, ESBL-E. coli belonging to group B2 accounted for only $8.6 \%$ of all ESBL-E. coli isolates from the food samples. This order of the most to least prevalent phylogenetic groups observed in the present study was similar to that observed in a recent study conducted in Nha Trang, Vietnam (Le et al. 2015). In addition, a similar phylogenetic profile was observed for ESBL-E. coli isolates from chicken samples in the Netherlands, with the highest percentage (44 \%) of isolates belonging to group B1, followed by groups A ( $28 \%$ ), D (23\%), and B2 (2 \%) (Kluytmans et al. 2013). Therefore, studies performed in the Netherlands and other regions of Vietnam showed that the prevalence of ESBL-E. coli isolates belonging to group B2 was significantly lower than the prevalence of ESBL-E. coli isolates belonging to other phylogenetic groups. However, the results of the present study showed a much higher prevalence of ESBL-E. coli isolates belonging to group B2 than reported in previous studies performed in other areas of Vietnam or in other countries. Since group B2 usually includes bacteria isolated from humans (Jakobsen et al. 2010), our findings imply a close relationship between ESBL-E. coli isolates from food samples and isolates from humans.
A high percentage (89.2 \%) of the ESBL-E. coli isolates from the food samples tested in this study showed MDR. The prevalence of extensively MDR ESBL-E. coli was especially high in fishery products (56-62\% isolates). Quantitative and qualitative analysis of antibiotic-resistant bacteria in fishery products (Van et al. 2007a, 2007b; Le et al. 2015) showed that up to $61 \%$ of $E$. coli isolates exhibit MDR. The prevalence of aquaculture as well as the unique distribution method for fishery products in rural areas of Vietnam (described above) could lead to cross contamination with antibiotic-resistant bacteria, particularly in markets, resulting in a high prevalence of these bacteria.

Since the spread of carbapenem-resistant bacteria in the community is a threat to public health due to the increased risk of intractable infection, the isolation of MEM-resistant ESBL-E. coli from pork samples is notable. Even though only one strain was isolated from the food samples tested in this study, possible dissemination of these bacteria in food should be monitored. The high rate of quinolone resistance in ESBL-E. coli isolates from food is also important. The heavy use of quinolone antibiotics in agriculture may be responsible for this high prevalence.

Food contaminated with ESBL-producing bacteria is a potential source for widespread dissemination of these bacteria in humans (Lazarus et al. 2015). The results of our study, combined with data from previous studies in different regions of Vietnam and other countries, suggests that rural areas are major contributors to the dissemination of not only ESBL-E. coli but also extensively MDR bacteria. Rural areas with livestock and aquaagriculture activities are one of the largest reservoirs of MDR bacteria and are therefore a major threat to public health. Thus, the development of stringent monitoring strategies and the promotion of hygienic food distribution practices are needed to control the spread of these antibiotic-resistant bacteria.

\section{Conclusions}

This study provided evidence of widespread dissemination of ESBL-E. coli in both livestock and fishery products obtained from a rural area in Vietnam. The high percentage of extensively MDR ESBL- $E$. coli in these food products highlights the importance of continuous monitoring for these bacteria in food distribution, particularly in underdeveloped countries.

\section{Abbreviations}

ESBL: extended-spectrum $\beta$-lactamase; MDR: multi-drug resistance; PCR: polymerase chain reaction; AMP: ampicillin; FOX: cefoxitin;

CTX: cefotaxime; CAZ: ceftazidime; MEM: meropenem; NAL: nalidixic acid; CIP: ciprofloxacin; KAN: kanamycin; STR: streptomycin; GEN: gentamycin; TET: tetracycline; FOF: fosfomycin; CHL: chloramphenicol; SXT: trimethoprimsulfamethoxazole; IMViC tests: indole, methyl red, Voges Proskauer and citrate utilization tests. 


\section{Competing interests}

The authors declare that they have no competing interests.

\section{Authors' contributions}

HVL: acquisition of data, analysis and interpretation of data, drafting the manuscript; RK: study concept and design, acquisition of data; DTK: acquisition of data; HTT: acquisition of data; TNN: acquisition of data; KNP: acquisition of data; MJ: acquisition of data; YK: study concept and design; TN: study concept and design; SU: acquisition of data; YY: study concept and design, analysis and interpretation of data, drafting manuscript and critical evaluation of manuscript to ensure accuracy of content. All authors read and approved the final manuscript.

\section{Acknowledgements}

This work was supported by the Japan Agency for Medical Research and Development (AMED)/Japan International Cooperation Agency (JICA) as part of the Science and Technology Research Partnership for Sustainable Development (SATREPS) and the Vietnam Ministry of Health.

\section{Author details}

${ }^{1}$ Thai Binh University of Medicine and Pharmacy, Thai Binh, Vietnam. ${ }^{2}$ Osaka Prefectural Institute of Public Health, Osaka, Japan. ${ }^{3}$ Osaka University Global Collaboration Center, 2-7 Yamadaoka, Suita, Osaka 565-0871, Japan.

\section{Received: 7 August 2015 Accepted: 16 December 2015}

\section{Published online: 29 December 2015}

\section{References}

Agersø Y, Aarestrup FM, Pedersen K, Seyfarth AM, Struve T, Hasman H. Prevalence of extended-spectrum cephalosporinase (ESC)-producing Escherichia coli in Danish slaughter pigs and retail meat identified by selective enrichment and association with cephalosporin usage. J Antimicrob Chemother. 2012;67:582-8. doi:10.1093/jac/dkr507.

Börjesson S, Egervärn M, Lindblad M, Englund S. Frequent occurrence of extended-spectrum beta-lactamase- and transferable Ampc beta-lactamaseproducing Escherichia coli on domestic chicken meat in Sweden. Appl Environ Microbiol. 2013;79:2463-6. doi:10.1128/AEM.03893-12.

Bush K, Jacoby GA. Updated functional classification of $\beta$-lactamases. Antimicrob Agents Chemother. 2010;54:969-76. doi:10.1128/AAC.01009-09.

Cantón R, Ruiz-Garbajosa P. Co-resistance: an opportunity for the bacteria and resistance genes. Curr Opin Pharmacol. 2011;11:477-85. doi:10.1016/ j.coph.2011.07.007.

Chen LF, Freeman JT, Nicholson B, Keiger A, Lancaster S, Joyce M, et al. Widespread dissemination of CTX-M-15 genotype extended-spectrum- $\beta$ lactamase-producing enterobacteriaceae among patients presenting to community hospitals in the southeastern United States. Antimicrob Agents Chemother. 2014:58:1200-2. doi:10.1128/AAC.01099-13.

Clinical and Laboratory Standards Institute. Performance standards for antimicrobial disk susceptibility tests; 21th informational supplement M100-S23. Wayne, PA: CLSI; 2013.

Coque TM, Baquero F, Cantón R. Increasing prevalence of ESBL-producing Enterobacteriaceae in Europe. Euro Surveill. 2008;13:47.

Cortés P, Blanc V, Mora A, Dahbi G, Blanco JE, Blanco M, et al. Isolation and characterization of potentially pathogenic antimicrobial-resistant Escherichia coli strains from chicken and pig farms in Spain. Appl Environ Microbiol. 2010;76:2799-805. doi:10.1128/AEM.02421-09.

Geser N, Stephan R, Hächler H. Occurrence and characteristics of extended-spectrum $\beta$-lactamase (ESBL) producing Enterobacteriaceae in food producing animals, minced meat and raw milk. BMC Vet Res. 2012;8:21. doi:10.1186/1746-6148-8-21.

Hawkey PM, Jones AM. The changing epidemiology of resistance. J Antimicrob Chemother. 2009;64 Suppl 1:i3-i10. doi:10.1093/jac/dkp256.

Hoa PTP, Managaki S, Nakada N, Nakada N, Takada H, Shimizu A, et al. Antibiotic contamination and occurrence of antibiotic-resistant bacteria in aquatic environments of northern Vietnam. Sci Total Environ. 2011;409:2894-901. doi:10.1016/..scitotenv.2011.04.030.

Jakobsen L, Kurbasic A, Skjøt-Rasmussen L, Ejrnaes K, Porsbo LJ, Pedersen K, et al. Escherichia coli isolates from broiler chicken meat, broiler chickens, pork, and pigs share phylogroups and antimicrobial resistance with communitydwelling humans and patients with urinary tract infection. Foodborne Pathog Dis. 2010;7:537-47. doi:10.1089/fpd.2009.0409.
Kluytmans JAJW, Overdevest ITMA, Willemsen I, Kluytmans-van den Bergh MFQ, van der Zwaluw K, Heck M, et al. Extended-spectrum $\beta$-lactamase-producing Escherichia coli from retail chicken meat and humans: comparison of strains, plasmids, resistance genes, and virulence factors. Clin Infect Dis. 2013;56:478-87. doi:10.1093/cid/cis929.

Lazarus B, Paterson DL, Mollinger $\mathrm{J}$, Rogers BA. Do human extraintestinal Escherichia coli infections resistant to expanded-spectrum cephalosporins originate from food-producing animals? A systematic review. Clin Infect Dis. 2015;60:439-52. doi:10.1093/cid/ciu785.

Le QP, Ueda S, Nguyen TNH, Dao TVK, Van Hoang TA, Tran TTN, et al. Characteristics of extended-spectrum $\beta$-lactamase-producing Escherichia coli in retail meats and shrimp at a local market in Vietnam. Foodborne Pathog Dis. 2015;12:719-25. doi:10.1089/fpd.2015.1954.

Leverstein-van Hall MA, Dierikx CM, Cohen Stuart J, Voets GM, van den Munckhof MP, van Essen-Zandbergen A, et al. Dutch patients, retail chicken meat and poultry share the same ESBL genes, plasmids and strains. Clin Microbiol Infect. 2011;17:873-80. doi:10.1111/j.1469-0691.2011.03497.x.

Liebana E, Carattoli A, Coque TM, Hasman H, Magiorakos A-P, Mevius D, et al. Public health risks of enterobacterial isolates producing extended-spectrum $\beta$-lactamases or AmpC $\beta$-lactamases in food and food-producing animals: an EU perspective of epidemiology, analytical methods, risk factors, and control options. Clin Infect Dis. 2013;56:1030-7. doi:10.1093/cid/cis1043.

Luvsansharav U-O, Hirai I, Nakata A, Imura K, Yamauchi K, Niki M, et al. Prevalence of and risk factors associated with faecal carriage of CTX-M B-lactamaseproducing Enterobacteriaceae in rural Thai communities. J Antimicrob Chemother. 2012:67:1769-74 doi:10.1093/jac/dks118.

Nakayama T, Ueda S, Huong BTM, Tuyen LD, Komalamisra C, Kusolsuk T, et al. Wide dissemination of extended-spectrum $\beta$-lactamase-producing Escherichia coli in community residents in the Indochinese peninsula. Infect Drug Resist. 2015;8:1-5. doi:10.2147/IDR.S74934.

Overdevest I, Willemsen I, Rijnsburger M, Eustace A, Xu L, Hawkey P, et al. Extended-spectrum $\beta$-lactamase genes of Escherichia coli in chicken meat and humans, The Netherlands. Emerg Infect Dis. 2011;17:1216-22. doi:10.3201/eid1707.110209.

Pitout JDD, Laupland KB. Extended-spectrum beta-lactamase-producing Enterobacteriaceae: an emerging public-health concern. Lancet Infect Dis. 2008:8:159-66. doi:10.1016/S1473-3099(08)70041-0.

Sasaki T, Hirai I, Niki M, Komalamisra C, Maipanich W, Kusolsuk T, et al. High prevalence of CTX-M B-lactamase-producing Enterobacteriaceae in stool specimens obtained from healthy individuals in Thailand. J Antimicrob Chemother. 2010;65:666-8. doi:10.1093/jac/dkq008.

Suzuki S, Hoa PTP. Distribution of quinolones, sulfonamides, tetracyclines in aquatic environment and antibiotic resistance in Indochina. Front Microbiol. 2012;3:67. doi:10.3389/fmicb.2012.00067.

Thampuran N, Surendraraj A, Surendran PK. Prevalence and characterization of typical and atypical Escherichia coli from fish sold at retail in Cochin, India. J Food Prot. 2005;68:2208-11.

Tian SF, Chen BY, Chu YZ, Wang S. Prevalence of rectal carriage of extendedspectrum beta-lactamase-producing Escherichia coli among elderly people in community settings in China. Can J Microbiol. 2008:54:781-5. doi:10.1139/w08-059.

Van TTH, Moutafis G, Istivan T, Tran LT, Coloe PJ. Detection of Salmonella spp. in retail raw food samples from Vietnam and characterization of their antibiotic resistance. Appl Environ Microbiol. 2007a;73:6885-90. doi:10.1128/AEM.00972-07.

Van TTH, Moutafis G, Tran LT, Coloe PJ. Antibiotic resistance in food-borne bacterial contaminants in Vietnam. Appl Environ Microbiol. 2007b;73:7906-11. doi:10.1128/AEM.00973-07.

Woerther P-L, Burdet C, Chachaty E, Andremont A. Trends in human fecal carriage of extended-spectrum $\beta$-lactamases in the community: toward the globalization of CTX-M. Clin Microbiol Rev. 2013;26:744-58. doi:10.1128/CMR.00023-13. 